\title{
A Mysterious Tale of Cardiogenic Delirium Induced by COVID-19
}

\author{
Saquib Navid Siddiqui
}

\section{ABSTRACT}

\begin{abstract}
A novel corona virus (SARS-COV-2) started to spread around the world eventually being declared as a global pandemic crisis by WHO in March,2020. Total number of cases have reached to a staggering 140 million by 17th April, 2021 with sad demise of 4 million of the world population. A few number of patients appear to be suffering from cardiac conductive abnormalities have been noted to be associated with SARS-CoV-2. This case unfolds story of a cardiogenic delirium induced by COVID-19. It's a roller coaster journey of a 62-year -old gentleman presenting with acute delirium which was later found to be due to low brain tissue perfusion resulting from complete heart block. After ruling out all possible causes for the sudden development of complete heart block we came into conclusion of association of SARS-CoV-2 in the development of complete heart block which retrospectively lead the gentleman to become delirious.
\end{abstract}

Keywords: SARS-CoV-2, COVID-19, delirium, complete heart block, cardiogenic delirium.

\section{Case Presentation}

A 62-year-old male, usually fit and healthy with no comorbidities was brought to the accident and emergency triage with acute onset delirium. Collateral history from wife revealed he had no prior history of memory impairment. On further query she mentioned him suffering from nonproductive cough and intermittent low-grade fever which subsided with paracetamol in the preceding 2 days of hospital admission. He had a positive exposure history to COVID-19 patient as his daughter was positive for SARS-CoV-2 virus. On arrival he was delirious with an Abbreviated Mental Test (AMT) score of 1 out of 10. Physical examination was noted for bradycardia with a stable blood pressure, and clear bilateral breath sounds. He was not hypoxic. Neurological examination revealed no focal abnormalities apart from cognitive impairment. He had no neck rigidity. Bedside urine dip test excluded any evidence of urine infection. Initial electrocardiogram was noted for third-degree AV block with a heart rate of $37 \mathrm{BM}$ (Fig. 1). Chest X-ray was clear. Full blood count, C-reactive protein were unremarkable apart from lymphopenia (0.6). Liver, renal, and thyroid function test remained within normal limits. Vitamin B12 and folate levels came back as normal too. Later, he became hemodynamically unstable as his systolic blood pressure dropped down to $88 \mathrm{~mm} \mathrm{hg}$.

Cardiology team evaluated the patient, who was later taken to the cardiac catheterization lab for an emergent temporary transvenous pacemaker. His troponin was within normal limit. CT scan of his head revealed no acute changes. Transthoracic echocardiography showed normal biventricular wall motion with left ventricular ejection fraction $62 \%$. Recent ECG performed 2 weeks prior to the incident was also unremarkable (Normal sinus rhythm with heart rate of 88 beats/minute). He was not on any regular medications. Twist in the tale came when his COVID-19 swab results came back as positive. In view to rule out a possible COVID-19 encephalitis lumber puncture was performed which was found to be unremarkable. The patient was placed in isolation (droplet precautions) and subsequently underwent elective permanent pacemaker implantation. Electrocardiogram performed after the procedure revealed paced rhythm. He did well postoperatively and completely regained his cognitive function post pacemaker insertion. He displayed no further respiratory symptoms whilst his stay in the hospital with post procedure chest $\mathrm{x}$-ray showing clear lung fields and a presence of the implanted pacemaker (Fig. 2). He was discharged within 48 hours of pacemaker implantation without any further complications. Therefore, in retrospective analysis of the case we came into conclusion that the low heart rate due to complete heart block resulted in hypoperfusion to the brain tissues resulting in development of sudden onset delirium. On further quest to find the aetiology for the complete heart block we ruled out cardiac, medication, and all other possible causes of complete heart block. After ruling out all common causes we think that the development of complete heart block and him catching the SARS-CoV-2 virus cannot be a coincidence. 


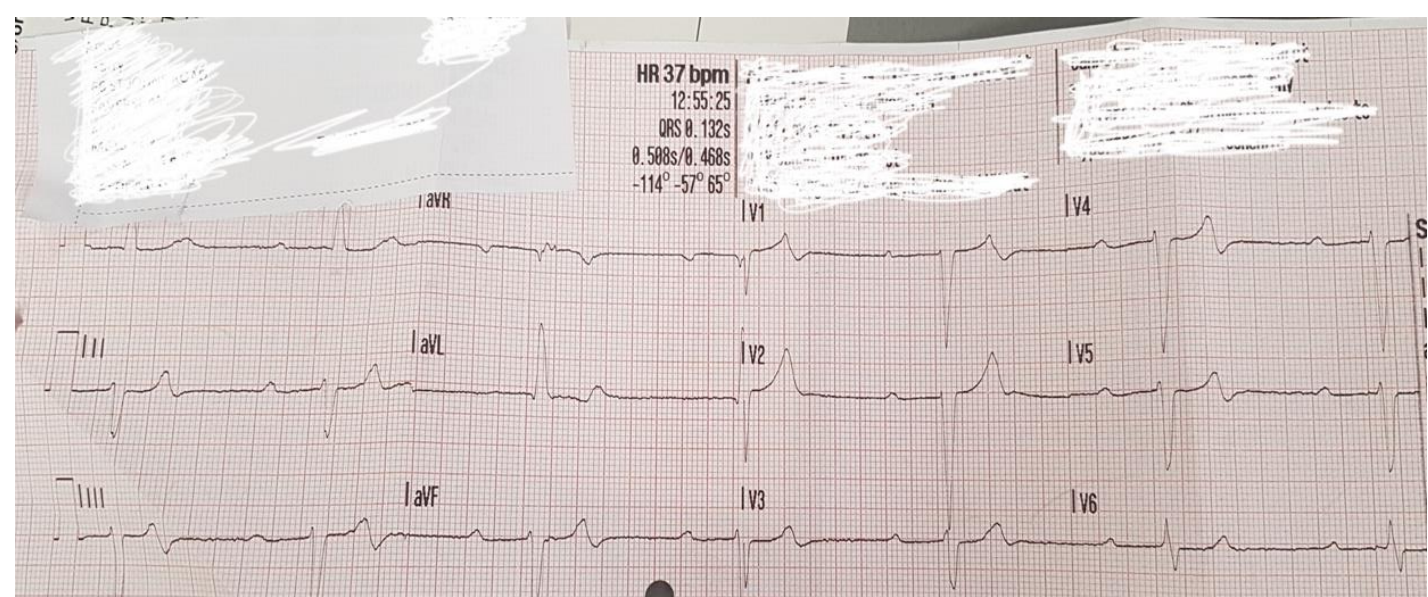

Fig. 1. ECG showing complete heart block.

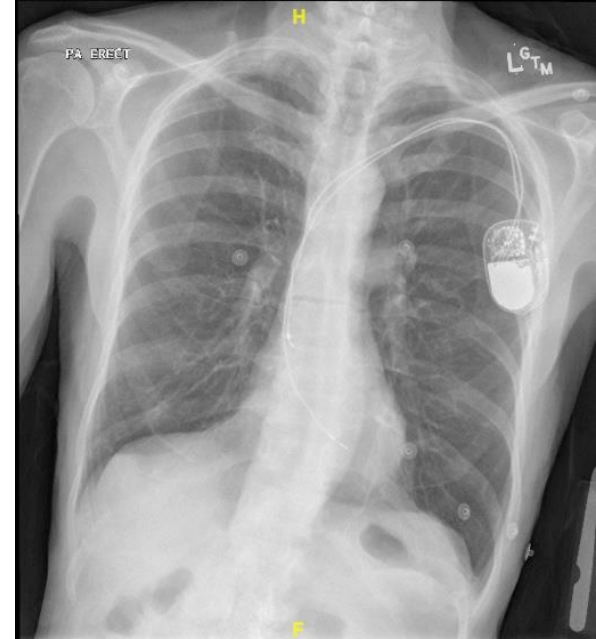

Fig. 2. Post pacemaker insertion CXR.

\section{DifFERENTIAL DiAGNOSES AND LABS}

Blood results showed marked lymphopenia at $0.6 \times 10^{9} / 1$ $\left(1.5-4.0 \times 10^{9} / 1\right)$. D-dimer, C-reactive protein, liver function, renal function, and thyroid function tests were unremarkable. Folate, vitamin B12 assay were normal. Lumber puncture was unremarkable. Initial electrocardiogram revealed complete heart block with heart rate of 37 beats/minute (Fig. 1). X-Ray chest post pacemaker insertion showed presence of pacemaker with elements of features of chronic obstructive pulmonary disease changes (Fig. 2). He had a positive swab test for SARS-CoV-2 by RT PCR method. Bedside urine dip stick was negative for leucocyte, nitrates. Urine routine microscopic examination too was unremarkable. CT head scan showed no acute changes. Transthoracic echocardiography showed normal biventricular wall motion with left ventricular ejection fraction $62 \%$.

\section{TREATMENT}

He had a pacemaker insertion during his hospital stays. He did not require any oxygen support as never displayed any respiratory symptoms whilst in the hospital. He was discharged within 48 hours of pacemaker insertion with cardiology outpatient follow up and was advised to selfisolate for 10 days.

\section{BACKGROUND \& DISCUSSION}

This case is unique in terms of its clinical presentation and corelating amongst multiple complex ongoing pathophysiological cascades induced by SARS-CoV-2 which potentially resulted in the development of delirium. After a thorough meticulous approach, we came into the conclusion that delirium onset resulted following hypo-perfusion insult to the brain caused by the low-rate secondary to complete heart block. After ruling out all possible causes for complete heart block we investigated recent studies and have found evidence of COVID-19 associated cardiac conduction defects along with development of complete heart block [1]. The etiopathologies of delirium is diverse and multifactorial. It ranges from acute stress responses due to medications, infection, inflammation, metabolic or toxic changes in the body to any brain lesion [2]. In our case we eliminated all potential risk factors for the delirium by ruling out urine infection, bacterial chest infection, COVID-19 encephalitis or any other form of bacterial/viral meningoencephalitis, metabolic or electrolyte abnormalities, medication or toxin, and stroke. He was not hypoxic which eliminates risk of hypoxic brain injury. The only abnormality we noticed was low heart rate due to sudden development of complete heart block which gives us the potential answer behind his sudden onset of delirium. We belief he developed extra-pulmonary manifestation of COVID-19 in the form of cardiac conduction defect (complete heart block) which eventually resulted in low brain tissue perfusion leading to cognitive abnormalities. Empirical evidence that cerebral hypoperfusion contributes to the development of delirium is limited possibly due to difficulties in measuring cerebral perfusion. Few studies have investigated hypoperfusion as a mechanism leading delirium. Smith and colleagues published their study findings which reveals poor cerebral perfusion is associated with cognitive impairment and delirium [3]. A low arterial oxygen saturation or decrease cerebral perfusion both can lead to brain injury. In our patient brain hypo-perfusion resulted from low heart rate. In general, cerebral autoregulatory reflexes ensure constant cerebral perfusion across a range of hemodynamic states so that brain tissue oxygenation should not correlate with hemodynamic variables, but loss of such autoregulation have been suggested in previous study results by Michael and colleagues [4]. Our hypothesis is SARS-CoV-2 resulted in 
loss of his autoregulatory mechanism; therefore, his brain perfusion became reliant on hemodynamic states which was compromised due to concomitant development of complete heart block. Our hypothesis proves it's worth as he regained his cognition fully within 24 hours of pacemaker implantation. Other aspect of our complex case is simultaneous occurrence of complete heart block. We managed to rule out all possible causes of complete heart block prior to the conclusion of a COVID-19 associated conduction defect. He was fit and healthy gentleman prior to hospital admission with no co-morbidities and not on any regular medications. His calcium, magnesium, phosphate, potassium levels were within normal limits. His thyroid function was unremarkable. No previous ischemic cardiac events and troponin was unremarkable during current hospital admission. No evidence of any other form of infection apart from SARS-CoV-2. Since the origin of COVID-19 the increasing number of cardiac manifestations induced by COVID-19 have generated great concern. Such patients are at risk of morbidity and mortality. A recent study of 138 patients hospitalized with COVID-19 infection revealed $16.7 \%$ and $7.2 \%$ patients later developed arrhythmia and acute cardiac injury, respectively [5]. Another study reveals $12.5 \%$ of patients with COVID-19 had evidence of myocardial injury with abnormalities which resembles myocarditis [6]. We are still at an early stage of understanding the mechanism of COVID-19 associated cardiac conductive defects; therefore, exact mechanism of SARS-CoV-2 virus induced complete heart block is yet to be clear. One potential theory is affinity of the coronavirus towards cells that express angiotensin-converting enzyme 2 (ACE2) protein which includes myocardial cells and initiation of the cytokines storm, mediated by abnormal $\mathrm{T}$ helper cells and hypoxiainduced high intracellular calcium resulting in cardiac myocyte apoptosis [7]. Such myocardial injury is associated with cardiac arrhythmia and even complete heart block [1], [8]. In a large survey by Gopinathannair found some incidences of complete heart block associated with COVID19 infection [9]. Another likely explanation for bradycardia and complete heart block associated with COVID-19 is release of pro-inflammatory cytokines (IL6, IL10, IL12, and TNF alpha) which targets cardiac pacemaker resulting in change of heart rate and rhythm [10]. To summarise cardiac conductive defects are a compilation of multi factorial components which includes viral myocardial damage, hypoxia, and increase release of pro-inflammatory cytokines [10].

In retrospective analysis of our complex case study, we can link the simultaneous development of complete heart block after being infected by SARS-CoV-2 resulting in low brain tissue perfusion eventually making him delirious which resolved after cardiac pacemaker implantation.

\section{LEARNING POINTS}

- Cardiac cause of delirium although are less frequent but can occure; specially in elderly population with cerebral cortical volume loss.

- SARS-CoV2 is associated is associated with cardiac conduction defects including complete heart block.
- If conduction abnormalities such as complete heart block do not resolve with resolution of viral illness will require cardiac intervention.

\section{CONCLUSION}

It requires a vigilent approach to rule out all possible causes of heart block prior to establishing link between SARS-CoV2 and heart block. As such cases have been rarely found we are not certain whether resolution of viral illness will correct the complete heart block but in our case as patient became haemodynamically unstable required a pacemaker insertion.

\section{ACKNOWLEDGMENT}

We would like to acknowledge our patient for permitting us to report the case for the benefit of medical science.

\section{REFERENCES}

[1] Azarkish M., Laleh Far V., Elamite M., et al. Transient complete heart block in a patient with critical COVID-19. Eur Heart J. 2020;41:2131.

[2] Fong T., G., Tulebaev S., R., Inouye S. K. Delirium in elderly adults: diagnosis, prevention and treatment. PMID: 19347026; doi: 10.1038/nrneurol.2009.24.

[3] Smith PJ, Blumenthal JA, Hoffman BM, et al. Reduced cerebral perfusion pressure worsens delirium outcomes following lung transplantation. Ann Am Thorac Soc. 2016;13. PMID: 26731642; doi: 10.1513/AnnalsATS.201507-454OC.

[4] Michael D. W., david M. M., john G. M., et al. Low brain tissue oxygenation contributes to the development of delirium in critically ill patients: A prospective observational study. PMID: 28668768; doi: 10.1016/j.jcrc.2017.06.009.

[5] Wang D, Hu B, Hu c, et al. Characteristics of 138 hospitalized patients with 2019 novel coronavirus-infected pneumonia in Wuhan,China.Jama.2020 Feb 7. Doi:10.1001/jama.2020.1585.

[6] Deng Q, Hu B, Zheng Y, et al. Suspected myocardial injury in patients with COVID-19: Evidence from front-line clinical observation in Wuhan, China. doi: 10.1016/j.ijcard.2020.03.087

[7] Oudit GY, Kassir Z, Jiang C, et al. SARS-coronavirus modulation of myocardial ACE2 expression and inflammation in patients with SARS. PMID: 19453650; doi: 10.1111/j.1365-2362.2009.02153.

[8] El Assad I., Hood-Pishchany M.I., Kheir J., et al. Complete heart block, severe ventricular dysfunction and myocardial inflammation in a child with COVID-19 infection. JACC Case Rep. 2020 PII:S26660849(20)30486-1.

[9] Gopinathannair R., Merchant F.M., Lakkireddy D.R., et al. COVID-19 and cardiac arrhythmias: a global perspective on arrhythmia characteristics and management strategies. J Interview Card Electrophysiology. 2020:1-8. Do I:10.1007/s10840-020-00789-9.

[10] Amaratunga E. A., Corbin D. S., Moran L., et al. Bradycardia in patients with COVID-19: A Calm Before the Storm? PMID: 32550090; doi: 10.7759/cereus.8599.

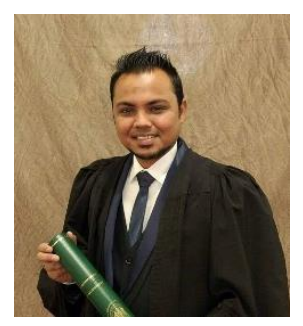

Dr. Saquib Navid Siddiqui completed MBBS in 2012 in Dhaka, Bangladesh. During his MBBS journey he obtained highest marks in all 3 professional examinations from his medical college remaining within top $15 \%$ of the total marks. Since then he underwent training in various sub-specialties of medicine in various tertiary level hospitals in Bangladesh. He completed MRCP (UK) in 2016 (One of the top scorer in MRCP paces exams from Bangladesh) and joined NHS in 2019 as trust grade specialist registrar in general medicine. He is currently working as a higher speciality trainee in respiratory medicine \& general medicine. He is the recipient of 3 prestigious awards while working for NHS (COVID-19 recognition award, Epic award, and COVID-19 appreciation award).

He has 5 other publications as the lead and corresponding author to his credit: 
1. 'A Unique Tale of COVID-19 induced concomitant overt disseminated intravascular coagulation and acute bilateral pulmonary embolism'published in International Journal Of Infectious Diseases on January, 2021.

2. 'Is standard Oral Dose Dexamethasone (6mg Once Daily) Prescribed For COVID-19 Pneumonitis Treating Autoimmune Haemolytic Anaemia Associated with Covid-19? A case Series Of Five Patients Providing Us with the answer' published in IJIRMS on Januray 2021.

3. 'A Rare Case Of Alcohol Intoxication Masquerading Cerebral Venous Sinus Thrombosis' published in EJMED on 20/11/2020.

4. 'A Case Of Massive Pulmonary Embolism in COVID-19 Pneumonitis' published in IOSR journal on 19/06/2020.

5. 'A Diagnostic Road To Damascus: A Case of Conversion to Pontine Infarct' published in IOSR journal on 10/07/2020.

$\mathrm{He}$ is an active contributor in providing education to the junior doctors of his trust by regularly participating in delivering various teaching sessions. $\mathrm{He}$ is also the initiator of a weekly teaching session in his previous trust (EKHUFT) in elderly care. He has completed formal course in teaching to improve his teaching skills. He has completed two qualitive improvement projects in his previous trust(EKHUFT) which has had profound influence in improving quality of providing health care service in the respective area (Quality improvement project on 'Evidence based clinical guidelines for the management of COVID-19 on the Oxford Ward High Dependency Unit' in 2020 and Quality improvement project on 'Prescription of Therapeutic Oxygen As Drug' in 2020). He has had 3 poster presentations in Society of Acute Medicine conference held in Glasgow, 2020. He has participated and completed various clinical courses and skill development programmes since starting his career in NHS 2019. In addition, he is actively working as associate regional advisor for the Royal College of Physicians of Edinburgh since May 2020. He is the lead author of Guidanace of use of CPAP in COVID-19 Pneumonitis which was accepted by the Gold command of his previous trust (EKHUFT) to be used in all patinets with hypoxic respiratory failure along with COVID-19 pneumonitis requiring CPAP support. He is a member of the British Thorasic Society, European Thorasic Society, and American College of Chest Physicians. 